\title{
On bounds involving $k$-Appell's hypergeometric functions
}

\author{
Muhammad Uzair Awan ${ }^{1 *}$, Muhammad Aslam Noor ${ }^{2,3}$, Marcela V Mihai ${ }^{4}$ and Khalida Inayat Noor ${ }^{3}$
}

\section{"Correspondence:}

awan.uzair@gmail.com

'Department of Mathematics,

Government College University,

Faisalabad, Pakistan

Full list of author information is

available at the end of the article

\begin{abstract}
In this paper, we derive a new extension of Hermite-Hadamard's inequality via $k$-Riemann-Liouville fractional integrals. Two new $k$-fractional integral identities are also derived. Then, using these identities as an auxiliary result, we obtain some new $k$-fractional bounds which involve $k$-Appell's hypergeometric functions. These bounds can be viewed as new $k$-fractional estimations of trapezoidal and mid-point type inequalities. These results are obtained for the functions which have the harmonic convexity property. We also discuss some special cases which can be deduced from the main results of the paper.
\end{abstract}

MSC: 26D15; 26A51; 33B15; 33C65

Keywords: convex functions; harmonic convex functions; $k$-fractional; $k$-Appell's hypergeometric functions; inequalities

\section{Introduction and preliminaries}

Convexity theory has played a pivotal role through its numerous applications in different fields of pure and applied sciences. In the past few years several new generalizations and extensions of classical convexity have been proposed in the literature, see [1-12]. Shi et al. [11] introduced the notion of harmonic convex sets as follows.

Definition 1.1 ([11]) A set $\Omega \subset \mathbb{R}_{+}$is said to be a harmonically convex set if

$$
\frac{x y}{t x+(1-t) y} \in \Omega, \quad \forall x, y \in \Omega, t \in[0,1] \text {. }
$$

Iscan [8] introduced the class of harmonic convex functions. The natural domain of harmonic convex functions is harmonic convex sets. Noor et al. [10] extended the definition of harmonic convex functions and defined a new generalization, which is called harmonic $h$-convex functions.

Definition 1.2 ([10]) Let $h:[0,1] \subseteq J \rightarrow \mathbb{R}$ be a real function. A function $f: \Omega \subset \mathbb{R}_{+} \rightarrow \mathbb{R}$ is said to be a harmonically $h$-convex function if

$$
f\left(\frac{x y}{t x+(1-t) y}\right) \leq h(1-t) f(x)+h(t) f(y), \quad \forall x, y \in I, t \in(0,1) .
$$

(c) The Author(s) 2017. This article is distributed under the terms of the Creative Commons Attribution 4.0 International License (http://creativecommons.org/licenses/by/4.0/), which permits unrestricted use, distribution, and reproduction in any medium, provided you give appropriate credit to the original author(s) and the source, provide a link to the Creative Commons license, and indicate if changes were made. 
Remark 1.3 Note that, if $h(t)=t, t^{s}, t^{-s}, t^{-1}$ and $t=1$, then the definition of harmonic $h$-convex functions reduces to the definitions of harmonic convex, harmonic $s$-convex, harmonic s-Godunova-Levin convex, harmonic Godunova-Levin and harmonic $P$-functions, respectively. Thus it is worth to mention here that the class of harmonic $h$-convex functions is quite unifying one as it naturally includes several other classes of harmonic convex functions.

Convexity theory has also a strong relationship with theory of inequalities, and resultantly many inequalities have been obtained via convex functions, see [6, 13-15]. Interested readers may find the importance of generalized convexity to variational inequalities and multiple objective optimization in [16-20]. One of the most extensively studied inequalities is Hermite-Hadamard's inequality. This inequality was proved by Hermite and Hadamard independently. It provides a necessary and sufficient condition for a function to be convex. Dragomir et al. [6] has written a nice monograph on Hermite-Hadamard type inequalities. Interested readers may find very interesting and useful details about these inequalities in this monograph. Khattri [21] discussed some very interesting applications of Hermite-Hadamard's inequality. Recently fractional calculus has attracted many researchers and thus become a powerful tool in many branches of mathematics. For some recent investigations in fractional calculus, see [22]. The classical form of RiemannLiouville integrals is defined as follows.

Definition 1.4 ([22]) Let $f \in L_{1}[a, b]$. Then the Riemann-Liouville integrals $J_{a^{+}}^{\alpha} f$ and $J_{b-}^{\alpha} f$ of order $\alpha>0$ with $a \geq 0$ are defined by

$$
J_{a^{+}}^{\alpha} f(x)=\frac{1}{\Gamma(\alpha)} \int_{a}^{x}(x-t)^{\alpha-1} f(t) \mathrm{d} t, \quad x>a
$$

and

$$
J_{b}^{\alpha} f(x)=\frac{1}{\Gamma(\alpha)} \int_{x}^{b}(t-x)^{\alpha-1} f(t) \mathrm{d} t, \quad x<b
$$

where

$$
\Gamma(\alpha)=\int_{0}^{\infty} e^{-t} x^{\alpha-1} \mathrm{~d} x
$$

is the well-known gamma function.

Sarikaya et al. [23] obtained Hermite-Hadamard type inequalities via Riemann-Liouville fractional integrals. Diaz et al. [24] introduced the generalized $k$-gamma function as

$$
\Gamma_{k}(x)=\lim _{n \rightarrow \infty} \frac{n ! k^{n}(n k)^{\frac{x}{k}-1}}{(x)_{n, k}}, \quad k>0, x \in \mathbb{C} \backslash k \mathbb{Z}^{-} .
$$

$\Gamma_{k}$ is one parameter deformation of the classical gamma function as $\Gamma_{k} \rightarrow \Gamma$ when $k \rightarrow 1$. $\Gamma_{k}$ is based on the repeated appearance of the expression of

$$
\phi(\phi+k)(\phi+2 k)(\phi+3 k) \cdots(\phi+(n-1) k) .
$$


This above statement is a function of the variable $\phi$ and is denoted by $(\phi)_{n, k}$. It is known as Pochhammer $k$-symbol, which reduces to classical Pochhammer symbol $(\phi)_{n}$ by taking $k=1$. The integral of $\Gamma_{k}$ is given by

$$
\Gamma_{k}(x)=\int_{0}^{\infty} t^{x-1} e^{-\frac{t^{k}}{k}} \mathrm{~d} t, \quad \Re(x)>0 .
$$

It is evident from (1.5) that

$$
\Gamma_{k}(x)=k^{\frac{x}{k}-1} \Gamma\left(\frac{x}{k}\right)
$$

Diaz et al. [24] also defined a $k$-beta function as

$$
\beta_{k}(x, y)=\frac{\Gamma_{k}(x) \Gamma_{k}(y)}{\Gamma_{k}(x+y)}, \quad \Re(x)>0, \Re(y)>0 .
$$

The integral form of a $k$-beta function is given by

$$
\beta_{k}(x, y)=\frac{1}{k} \int_{0}^{1} t^{\frac{x}{k}-1}(1-t)^{\frac{x}{k}-1} \mathrm{~d} t
$$

From (1.5) and (1.7) one can have

$$
\beta_{k}(x, y)=\frac{1}{k} \beta\left(\frac{x}{k}, \frac{y}{k}\right)
$$

Using these definitions of $k$-gamma and $k$-beta functions, Mubeen et al. [25] introduced the $k$-Riemann-Liouville fractional integral of the type

$$
{ }_{k} J^{\alpha} f(x)=\frac{1}{k \Gamma_{k}(\alpha)} \int_{0}^{x}(x-t)^{\frac{\alpha}{k}-1} f(t) \mathrm{d} t, \quad \alpha>0, x>0, k>0 .
$$

It is obvious that when $k \rightarrow 1$, the above definition reduces to classical Riemann-Liouville fractional integrals.

Sarikaya et al. [26] introduced the notion of $k$-Riemann-Liouville fractional integrals and discussed some of its interesting applications with respect to inequalities.

To be more precise, let $f$ be piecewise continuous on $I^{*}=(0, \infty)$ and integrable on any finite subinterval of $I=[0, \infty]$. Then, for $t>0$, we consider the $k$-Riemann-Liouville fractional integral of $f$ of order $\alpha$

$$
{ }_{k} J_{a}^{\alpha} f(x)=\frac{1}{k \Gamma_{k}(\alpha)} \int_{a}^{x}(x-t)^{\frac{\alpha}{k}-1} f(t) \mathrm{d} t, \quad x>a, k>0 .
$$

For more details, see [26]. Note that when $k \rightarrow 1, k$-Riemann-Liouville fractional integrals become classical Riemann-Liouville fractional integrals. It is worth mentioning here that the notion of $k$-Riemann-Liouville fractional integral is the significant generalization of all above Riemann-Liouville fractional integrals. We would like to emphasize that for $k \neq 1$ the properties of $k$-Riemann-Liouville fractional integrals are quite different from those 
of classical Riemann-Liouville fractional integrals. Due to these facts, the $k$-RiemannLiouville fractional integrals have important applications in several branches of pure and applied sciences, see [24, 26, 27].

The integral representation of $k$-Appell's series $F_{1, k}$, where $k>0$, is

$$
F_{1, k}=\frac{\Gamma_{k}(c)}{k \Gamma_{k}\left(a^{\prime}\right) \Gamma_{k}\left(c-a^{\prime}\right)} \int_{0}^{1} t^{\frac{a^{\prime}}{k}-1}(1-t)^{\frac{c-a^{\prime}}{k}}-1\left(1-k z_{1} t\right)^{-\frac{b_{1}}{k}}\left(1-k z_{2} t\right)^{-\frac{b_{2}}{k}} \mathrm{~d} t .
$$

For some more details, see [27].

\section{Some new auxiliary results}

In this section, we derive some new $k$-fractional identities which will serve as auxiliary results for the developments of our next results.

Lemma 2.1 Let $f: I \backslash\{0\} \rightarrow \mathbb{R}$ be differentiable on $I^{\circ}$ such that $f^{\prime} \in L[a, b]$, where $a, b \in I$ with $a<b$, then

$$
T_{f}(a, b ; \alpha, k ; g)=\frac{a b(b-a)}{2} \int_{0}^{1} \frac{\left[t^{\frac{\alpha}{k}}-(1-t)^{\frac{\alpha}{k}}\right]}{[t a+(1-t) b]^{2}} f^{\prime}\left(\frac{a b}{t a+(1-t) b}\right) \mathrm{d} t
$$

where

$$
\begin{aligned}
& T_{f}(a, b ; \alpha, k ; g) \\
& \quad=\frac{f(a)+f(b)}{2}-\frac{\Gamma_{k}(\alpha+k)}{2}\left(\frac{a b}{b-a}\right)^{\frac{\alpha}{k}}\left[{ }_{k} J_{\frac{1}{b^{+}}}^{\alpha}(f \circ g)\left(\frac{1}{a}\right)+{ }_{k} J_{\frac{1}{a^{-}}}^{\alpha}(f \circ g)\left(\frac{1}{b}\right)\right] .
\end{aligned}
$$

Proof It suffices to show that

$$
\begin{aligned}
T_{f}(a, b ; \alpha, k ; g) & =\frac{a b(b-a)}{2} \int_{0}^{1} \frac{\left[t^{\frac{\alpha}{k}}-(1-t)^{\frac{\alpha}{k}}\right]}{[t a+(1-t) b]^{2}} f^{\prime}\left(\frac{a b}{t a+(1-t) b}\right) \mathrm{d} t \\
& =K_{1}+K_{2} .
\end{aligned}
$$

Now integrating by parts yields

$$
\begin{aligned}
K_{1} & =\frac{a b(b-a)}{2} \int_{0}^{1} \frac{t^{\frac{\alpha}{k}}}{[t a+(1-t) b]^{2}} f^{\prime}\left(\frac{a b}{t a+(1-t) b}\right) \mathrm{d} t \\
& =\frac{1}{2}\left[f(b)-\frac{k \Gamma_{k}(\alpha+k)}{k}\left(\frac{a b}{b-a}\right)^{\frac{\alpha}{k}} \frac{1}{k \Gamma_{k}(\alpha)} \int_{\frac{1}{b}}^{\frac{1}{a}}\left(\frac{1}{a}-x\right)^{\frac{\alpha}{k}-1} f\left(\frac{1}{x}\right) \mathrm{d} x\right] \\
& =\frac{f(b)}{2}-\frac{\Gamma_{k}(\alpha+k)}{2}\left(\frac{a b}{b-a}\right)^{\frac{\alpha}{k}} J_{\frac{1}{b^{+}}}^{\alpha}(f \circ g)\left(\frac{1}{a}\right) .
\end{aligned}
$$

Similarly

$$
\begin{aligned}
K_{2} & =\frac{a b(b-a)}{2} \int_{0}^{1} \frac{(1-t)^{\frac{\alpha}{k}}}{[t a+(1-t) b]^{2}} f^{\prime}\left(\frac{a b}{t a+(1-t) b}\right) \mathrm{d} t \\
& =\frac{f(a)}{2}-\frac{\Gamma_{k}(\alpha+k)}{2}\left(\frac{a b}{b-a}\right)^{\frac{\alpha}{k}} J_{\frac{1}{a^{-}}}^{\alpha}(f \circ g)\left(\frac{1}{b}\right) .
\end{aligned}
$$


Combining (2.1), (2.2) and (2.3) completes the proof.

Lemma 2.2 Under the assumptions of Lemma 2.1 and $k=1$, we have

$$
T_{f}(a, b ; \alpha, 1 ; g)=\frac{a b(b-a)}{2} \int_{0}^{1} \frac{t^{\alpha}-(1-t)^{\alpha}}{[t a+(1-t) b]^{2}} f^{\prime}\left(\frac{a b}{t a+(1-t) b}\right) \mathrm{d} t
$$

where

$$
T_{f}(a, b ; \alpha, 1 ; g)=\frac{f(a)+f(b)}{2}-\frac{\Gamma(\alpha+1)}{2}\left(\frac{a b}{b-a}\right)^{\alpha}\left[J_{\frac{1}{b^{+}}}^{\alpha}(f \circ g)\left(\frac{1}{a}\right)+J_{\frac{1}{a^{-}}}^{\alpha}(f \circ g)\left(\frac{1}{b}\right)\right] .
$$

This is due to Iscan [8].

Lemma 2.3 Let $f: I \backslash\{0\} \rightarrow \mathbb{R}$ be differentiable on $I^{\circ}$ such that $f^{\prime} \in L[a, b]$, where $a, b \in I$ with $a<b$, then

$$
\begin{aligned}
M_{f}(a, b ; \alpha, k ; g)= & \frac{1}{2} \sum_{i=1}^{3} I_{i} \\
= & \frac{1}{2}\left[a b(b-a) \int_{0}^{\frac{1}{2}} \frac{1}{[t a+(1-t) b]^{2}} f^{\prime}\left(\frac{a b}{t a+(1-t) b}\right) \mathrm{d} t\right. \\
& -a b(b-a) \int_{\frac{1}{2}}^{1} \frac{1}{[t a+(1-t) b]^{2}} f^{\prime}\left(\frac{a b}{t a+(1-t) b}\right) \mathrm{d} t \\
& \left.-a b(b-a) \int_{0}^{1}\left[(1-t)^{\frac{\alpha}{k}}-t^{\frac{\alpha}{k}}\right] \frac{1}{[t a+(1-t) b]^{2}} f^{\prime}\left(\frac{a b}{t a+(1-t) b}\right) \mathrm{d} t\right],
\end{aligned}
$$

where

$$
\begin{aligned}
& M_{f}(a, b ; \alpha, k ; g) \\
& \quad=f\left(\frac{2 a b}{a+b}\right)-\frac{\Gamma_{k}(\alpha+1)}{2}\left(\frac{a b}{b-a}\right)^{\frac{\alpha}{k}}\left\{{ }_{k} J_{\frac{1}{b^{-}}}^{\alpha}(f \circ g)\left(\frac{1}{a}\right)+{ }_{k} J_{\frac{1}{a^{+}}}^{\alpha}(f \circ g)\left(\frac{1}{b}\right)\right\} .
\end{aligned}
$$

Proof Calculate $I_{1}, I_{2}$ and $I_{3}$ as follows:

$$
\begin{aligned}
I_{1} & =a b(b-a) \int_{0}^{\frac{1}{2}} \frac{1}{[t a+(1-t) b]^{2}} f^{\prime}\left(\frac{a b}{t a+(1-t) b}\right) \mathrm{d} t \\
& =f\left(\frac{2 a b}{a+b}\right)-f(a) .
\end{aligned}
$$

Now

$$
\begin{aligned}
I_{2} & =-a b(b-a) \int_{\frac{1}{2}}^{1} \frac{1}{[t a+(1-t) b]^{2}} f^{\prime}\left(\frac{a b}{t a+(1-t) b}\right) \mathrm{d} t \\
& =f\left(\frac{2 a b}{a+b}\right)-f(b) .
\end{aligned}
$$


Also

$$
\begin{aligned}
I_{3} & =-a b(b-a) \int_{0}^{1}\left[(1-t)^{\frac{\alpha}{k}}-t^{\frac{\alpha}{k}}\right] \frac{1}{[t a+(1-t) b]^{2}} f^{\prime}\left(\frac{a b}{t a+(1-t) b}\right) \mathrm{d} t \\
& =-\int_{0}^{1}\left[(1-t)^{\frac{\alpha}{k}}-t^{\frac{\alpha}{k}}\right] \mathrm{d} f\left(\frac{a b}{t a+(1-t) b}\right) \\
& =-\int_{0}^{1}(1-t)^{\frac{\alpha}{k}} \mathrm{~d} f\left(\frac{a b}{t a+(1-t) b}\right)+\int_{0}^{1} t^{\frac{\alpha}{k}} \mathrm{~d} f\left(\frac{a b}{t a+(1-t) b}\right) \\
& =I_{I}+I_{I I} .
\end{aligned}
$$

Now consider

$$
\begin{aligned}
I_{I} & =-\int_{0}^{1}(1-t)^{\frac{\alpha}{k}} \mathrm{~d} f\left(\frac{a b}{t a+(1-t) b}\right) \\
& =f(a)-\frac{\alpha}{k} \int_{0}^{1}(1-t)^{\frac{\alpha}{k}-1} f\left(\frac{a b}{t a+(1-t) b}\right) \mathrm{d} t .
\end{aligned}
$$

Now suppose $u=\frac{a b}{t a+(1-t) b}$, then

$$
I_{I}=f(a)-\frac{\alpha}{k}\left(\frac{a b}{b-a}\right)^{\frac{\alpha}{k}} \int_{a}^{b}\left(\frac{1}{u}-\frac{1}{b}\right)^{\frac{\alpha}{k}-1} \frac{1}{u^{2}} f(u) \mathrm{d} u
$$

Again suppose $u=\frac{1}{t}$, then

$$
I_{I}=f(a)-\Gamma_{k}(\alpha+k)\left(\frac{a b}{b-a}\right)^{\frac{\alpha}{k}}{ }_{\frac{1}{b^{-}}}^{\alpha}(f \circ g)\left(\frac{1}{a}\right) .
$$

Similarly

$$
I_{I I}=f(b)-\Gamma_{k}(\alpha+k)\left(\frac{a b}{b-a}\right)^{\frac{\alpha}{k}}{ }_{k}^{\alpha} \frac{1}{a^{+}}(f \circ g)\left(\frac{1}{b}\right) .
$$

Using (2.7) and (2.8) in (2.6) and then adding the resultant with (2.4) and (2.5) completes the proof.

Lemma 2.4 Under the assumptions of Lemma 2.3 , if $k \rightarrow 1$, we have

$$
\begin{aligned}
M_{f}(a, b ; \alpha, 1 ; g)= & \frac{1}{2} \sum_{i=1}^{3} I_{i} \\
= & \frac{1}{2}\left[a b(b-a) \int_{0}^{\frac{1}{2}} \frac{1}{[t a+(1-t) b]^{2}} f^{\prime}\left(\frac{a b}{t a+(1-t) b}\right) \mathrm{d} t\right. \\
& -a b(b-a) \int_{\frac{1}{2}}^{1} \frac{1}{[t a+(1-t) b]^{2}} f^{\prime}\left(\frac{a b}{t a+(1-t) b}\right) \mathrm{d} t \\
& \left.-a b(b-a) \int_{0}^{1}\left[(1-t)^{\alpha}-t^{\alpha}\right] \frac{1}{[t a+(1-t) b]^{2}} f^{\prime}\left(\frac{a b}{t a+(1-t) b}\right) \mathrm{d} t\right]
\end{aligned}
$$


Awn et al. Journal of Inequalities and Applications (2017) 2017:118

Page 7 of 15

where

$$
M_{f}(a, b ; \alpha, 1 ; g)=f\left(\frac{2 a b}{a+b}\right)-\frac{\Gamma(\alpha+1)}{2}\left(\frac{a b}{b-a}\right)^{\frac{\alpha}{k}}\left\{J_{\frac{1}{b^{-}}}^{\alpha}(f \circ g)\left(\frac{1}{a}\right)+J_{\frac{1}{a^{+}}}^{\alpha}(f \circ g)\left(\frac{1}{b}\right)\right\} \text {. }
$$

This result is due to Set et al. [28].

3 Results and discussions

In this section, we derive some new $k$-fractional integral inequalities.

Theorem 3.1 Let $f: I \backslash\{0\} \rightarrow \mathbb{R}$ be a harmonically h-convex function where $a, b \in I$ with $a<b$. If $\in L[a, b]$, then, for $h\left(\frac{1}{2}\right) \neq 0$, we have

$$
\begin{aligned}
\frac{k}{\alpha h\left(\frac{1}{2}\right)} f\left(\frac{2 a b}{a+b}\right) & \leq\left(\frac{a b}{b-a}\right)^{\frac{\alpha}{k}} k \Gamma_{k}(\alpha)\left\{{ }_{k} J_{\frac{1}{a^{-}}}^{\alpha}(f \circ g)\left(\frac{1}{b}\right)+{ }_{k} J_{\frac{1}{b^{+}}}^{\alpha}(f \circ g)\left(\frac{1}{a}\right)\right\} \\
& \leq[f(a)+f(b)] \int_{0}^{1} t^{\frac{\alpha}{k}-1}[h(1-t)+h(t)] \mathrm{d} t .
\end{aligned}
$$

Proof Since $f$ is a harmonically $h$-convex function, so we have

$$
f\left(\frac{2 a b}{(1-t) a+t b}\right) \leq h\left(\frac{1}{2}\right)\left[f\left(\frac{a b}{t a+(1-t) b}\right)+f\left(\frac{a b}{(1-t) a+t b}\right)\right] .
$$

Multiplying both sides of the above inequality by $t^{\frac{\alpha}{k}-1}$ and integrating it with respect to $t$ on $[0,1]$, we have

$$
\begin{aligned}
& \frac{k}{\alpha} f\left(\frac{2 a b}{a+b}\right) \\
& \quad=f\left(\frac{2 a b}{a+b}\right) \int_{0}^{1} t^{\frac{\alpha}{k}-1} \mathrm{~d} t \\
& \quad \leq h\left(\frac{1}{2}\right)\left[\int_{0}^{1} t^{\frac{\alpha}{k}-1} f\left(\frac{a b}{t a+(1-t) b}\right) \mathrm{d} t+\int_{0}^{1} t^{\frac{\alpha}{k}-1} f\left(\frac{a b}{(1-t) a+t b}\right) \mathrm{d} t\right] \\
& =h\left(\frac{1}{2}\right)\left(\frac{a b}{b-a}\right)^{\frac{\alpha}{k}}\left\{\int_{\frac{1}{b}}^{\frac{1}{a}}\left(x-\frac{1}{b}\right)^{\frac{\alpha}{k}-1} f\left(\frac{1}{x}\right) \mathrm{d} x+\int_{\frac{1}{b}}^{\frac{1}{a}}\left(\frac{1}{a}-x\right)^{\frac{\alpha}{k}-1} f\left(\frac{1}{x}\right) \mathrm{d} x\right\} \\
& =h\left(\frac{1}{2}\right)\left(\frac{a b}{b-a}\right)^{\frac{\alpha}{k}} k \Gamma_{k}(\alpha)\left\{{ }_{k} J_{\frac{1}{a^{-}}}^{\alpha}(f \circ g)\left(\frac{1}{b}\right)+{ }_{k} J_{\frac{1}{b^{+}}}^{\alpha}(f \circ g)\left(\frac{1}{a}\right)\right\} .
\end{aligned}
$$

This implies

$$
\frac{k}{\alpha h\left(\frac{1}{2}\right)} f\left(\frac{2 a b}{a+b}\right) \leq\left(\frac{a b}{b-a}\right)^{\frac{\alpha}{k}} k \Gamma_{k}(\alpha)\left\{{ }_{k} J_{\frac{1}{a^{-}}}^{\alpha}(f \circ g)\left(\frac{1}{b}\right)+{ }_{k} J_{\frac{1}{b^{+}}}^{\alpha}(f \circ g)\left(\frac{1}{a}\right)\right\} .
$$

Now

$$
\begin{aligned}
& f\left(\frac{a b}{t a+(1-t) b}\right) \leq h(1-t) f(a)+h(t) f(b), \\
& f\left(\frac{a b}{(1-t) a+t b}\right) \leq h(t) f(a)+h(1-t) f(b) .
\end{aligned}
$$


Adding the above two inequalities and multiplying both sides by $t^{\frac{\alpha}{k}-1}$, we have

$$
t^{\frac{\alpha}{k}-1} f\left(\frac{a b}{t a+(1-t) b}\right)+t^{\frac{\alpha}{k}-1} f\left(\frac{a b}{(1-t) a+t b}\right) \leq t^{\frac{\alpha}{k}-1}[h(1-t)+h(t)][f(a)+f(b)] .
$$

Integrating the above inequality with respect to $t$ on $[0,1]$, we have

$$
\begin{aligned}
& \left(\frac{a b}{b-a}\right)^{\frac{\alpha}{k}} k \Gamma_{k}(\alpha)\left\{{ }_{k} J_{\frac{1}{a^{-}}}^{\alpha}(f \circ g)\left(\frac{1}{b}\right)+{ }_{k} J_{\frac{1}{b^{+}}}^{\alpha}(f \circ g)\left(\frac{1}{a}\right)\right\} \\
& \leq[f(a)+f(b)] \int_{0}^{1} t^{\frac{\alpha}{k}-1}[h(1-t)+h(t)] \mathrm{d} t .
\end{aligned}
$$

Summing inequalities (3.1) and (3.2) completes the proof.

We now discuss some special cases of Theorem 3.1.

I. If $h(t)=t$ in Theorem 3.1, then we have the following new result.

Corollary 3.2 Let $f: I \backslash\{0\} \rightarrow \mathbb{R}$ be a harmonically convex function, where $a, b \in I$ with $a<b$. Iff $\in L[a, b]$, then we have

$$
\begin{aligned}
\frac{2 k}{\alpha} f\left(\frac{2 a b}{a+b}\right) & \leq\left(\frac{a b}{b-a}\right)^{\frac{\alpha}{k}} k \Gamma_{k}(\alpha)\left\{{ }_{k} J_{\frac{1}{a^{-}}}^{\alpha}(f \circ g)\left(\frac{1}{b}\right)+{ }_{k} J_{\frac{1}{b^{+}}}^{\alpha}(f \circ g)\left(\frac{1}{a}\right)\right\} \\
& \leq \frac{k[f(a)+f(b)]}{\alpha} .
\end{aligned}
$$

II. If $h(t)=t^{s}$ in Theorem 3.1, then we have the following new result.

Corollary 3.3 Let $f: I \backslash\{0\} \rightarrow \mathbb{R}$ be a harmonically s-convex function, where $a, b \in I$ with $a<b$. If $\in L[a, b]$, then we have

$$
\begin{aligned}
\frac{2^{s} k}{\alpha} f\left(\frac{2 a b}{a+b}\right) & \leq\left(\frac{a b}{b-a}\right)^{\frac{\alpha}{k}} k \Gamma_{k}(\alpha)\left\{{ }_{k} J_{\frac{1}{a^{-}}}^{\alpha}(f \circ g)\left(\frac{1}{b}\right)+{ }_{k} J_{\frac{1}{b^{+}}}^{\alpha}(f \circ g)\left(\frac{1}{a}\right)\right\} \\
& \leq[f(a)+f(b)]\left(k B_{k}(\alpha, k(s+1))-\frac{k}{\alpha+k s}\right) .
\end{aligned}
$$

III. If $h(t)=t^{-s}$ in Theorem 3.1, then we have the following new result.

Corollary 3.4 Let $f: I \backslash\{0\} \rightarrow \mathbb{R}$ be a harmonically s-Godunova-Levin convex function, where $a, b \in I$ with $a<b$. If $f \in L[a, b]$, then, for $\alpha>k s$, we have

$$
\begin{aligned}
\frac{k}{2^{s} \alpha} f\left(\frac{2 a b}{a+b}\right) & \leq\left(\frac{a b}{b-a}\right)^{\frac{\alpha}{k}} k \Gamma_{k}(\alpha)\left\{{ }_{k} J_{\frac{1}{a^{-}}}^{\alpha}(f \circ g)\left(\frac{1}{b}\right)+{ }_{k} J_{\frac{1}{b^{+}}}^{\alpha}(f \circ g)\left(\frac{1}{a}\right)\right\} \\
& \leq[f(a)+f(b)]\left(k B_{k}(\alpha, k(1-s))-\frac{k}{\alpha-k s}\right) .
\end{aligned}
$$

IV. If $h(t)=1$ in Theorem 3.1, then we have the following new result. 
Corollary 3.5 Let $f: I \backslash\{0\} \rightarrow \mathbb{R}$ be a harmonic P-function, where $a, b \in I$ with $a<b$. If $f \in L[a, b]$, then we have

$$
\begin{aligned}
\frac{k}{\alpha} f\left(\frac{2 a b}{a+b}\right) & \leq\left(\frac{a b}{b-a}\right)^{\frac{\alpha}{k}} k \Gamma_{k}(\alpha)\left\{{ }_{k} J_{\frac{1}{a^{-}}}^{\alpha}(f \circ g)\left(\frac{1}{b}\right)+{ }_{k} J_{\frac{1}{b^{+}}}^{\alpha}(f \circ g)\left(\frac{1}{a}\right)\right\} \\
& \leq \frac{2 k[f(a)+f(b)]}{\alpha} .
\end{aligned}
$$

Now using the auxiliary results, we derive some trapezoidal and mid-point type inequalities.

Theorem 3.6 Assume that $f:[0,1] \rightarrow \mathbb{R}$ is a differentiable function such that $\left|f^{\prime}\right|^{q}$ is a harmonic convex function on $[0,1]$. Then

$$
\left|T_{f}(a, b ; \alpha, k ; g)\right| \leq \frac{a b(b-a)}{2} \cdot I^{1-\frac{1}{q}} \cdot J^{\frac{1}{q}},
$$

where

$$
I=\int_{0}^{1} \frac{\left|t^{\frac{\alpha}{k}}-(1-t)^{\frac{\alpha}{k}}\right|}{[t a+(1-t) b]^{2}} \mathrm{~d} t=\frac{1}{b^{2}}\left(I_{1}-\frac{1}{2^{\alpha / k}} I_{2}+I_{3}-I_{4}\right)
$$

with

$$
\begin{aligned}
& I_{1}=k F_{1, k}\left(k,-\alpha, 2 k, 2 k ; \frac{1}{2 k}, \frac{b-a}{2 b k}\right) ; \\
& I_{2}=k B_{k}(\alpha+k, 1) F_{1, k}\left(\alpha+k, 0,2 k, \alpha+k+1 ; 0, \frac{b-a}{2 b k}\right) ; \\
& I_{3}=k B_{k}(\alpha+k, 1) F_{1, k}\left(\alpha+k, 0,2 k, \alpha+k+1 ; 0, \frac{b-a}{b k}\right) ; \\
& I_{4}=k B_{k}(k, \alpha+k) F_{1, k}\left(k, 0,2 k, \alpha+2 k ; 0, \frac{b-a}{b k}\right),
\end{aligned}
$$

and

$$
\begin{aligned}
J & =\int_{0}^{1} \frac{\left|t^{\frac{\alpha}{k}}-(1-t)^{\frac{\alpha}{k}}\right|}{[t a+(1-t) b]^{2}}\left|f^{\prime}\left(\frac{a b}{t a+(1-t) b}\right)\right|^{q} \mathrm{~d} t \\
& \leq \frac{1}{b^{2}}\left[\left|f^{\prime}(a)\right|^{q}\left(J_{1}-\frac{1}{2^{\alpha / k}} J_{2}+J_{5}-J_{7}\right)+\left|f^{\prime}(b)\right|^{q}\left(\frac{1}{2} J_{3}-\frac{1}{2^{\alpha / k+1}} J_{4}+J_{6}-J_{8}\right)\right],
\end{aligned}
$$

with

$$
\begin{aligned}
& J_{1}=k B_{k}(k, k) F_{1, k}\left(k,-\alpha-k, 2 k, 2 k ; \frac{1}{2 k}, \frac{b-a}{2 b k}\right) ; \\
& J_{2}=k B_{k}(\alpha+k, k) F_{1, k}\left(\alpha+k,-k, 2 k, \alpha+2 k ; \frac{1}{2 k}, \frac{b-a}{2 b k}\right) ; \\
& J_{3}=k B_{k}(2 k, k) F_{1, k}\left(2 k,-\alpha, 2 k, 3 k ; \frac{1}{2 k}, \frac{b-a}{2 b k}\right) ; \\
& J_{4}=k B_{k}(\alpha+2 k, k) F_{1, k}\left(\alpha+2 k, 0,2 k, \alpha+3 k ; 0, \frac{b-a}{2 b k}\right) ;
\end{aligned}
$$




$$
\begin{aligned}
& J_{5}=k B_{k}(\alpha+k, 2 k) F_{1, k}\left(\alpha+k, 0,2 k, \alpha+3 k ; 0, \frac{b-a}{b k}\right) ; \\
& J_{6}=k B_{k}(\alpha+2 k, k) F_{1, k}\left(\alpha+2 k, 0,2 k, \alpha+3 k ; 0, \frac{b-a}{b k}\right) ; \\
& J_{7}=k B_{k}(k, \alpha+2 k) F_{1, k}\left(k, 0,2 k, \alpha+3 k ; 0, \frac{b-a}{b k}\right) \\
& J_{8}=k B_{k}(2 k, \alpha+k) F_{1, k}\left(2 k, 0,2 k, \alpha+3 k ; 0, \frac{b-a}{b k}\right) .
\end{aligned}
$$

Proof From Lemma 2.1, using the property of modulus and the power-mean inequality, we have

$$
\begin{aligned}
& \left|T_{f}(a, b ; \alpha, k ; g)\right| \\
& \quad=\left|\frac{a b(b-a)}{2} \int_{0}^{1} \frac{\left[t^{\frac{\alpha}{k}}-(1-t)^{\frac{\alpha}{k}}\right]}{[t a+(1-t) b]^{2}} f^{\prime}\left(\frac{a b}{t a+(1-t) b}\right) \mathrm{d} t\right| \\
& \quad \leq \frac{a b(b-a)}{2} \int_{0}^{1} \frac{\left|t^{\frac{\alpha}{k}}-(1-t)^{\frac{\alpha}{k}}\right|}{[t a+(1-t) b]^{2}}\left|f^{\prime}\left(\frac{a b}{t a+(1-t) b}\right)\right| \mathrm{d} t \\
& \quad \leq \frac{a b(b-a)}{2} I^{1-\frac{1}{q}} J^{\frac{1}{q}},
\end{aligned}
$$

where

$$
\begin{aligned}
I & =\int_{0}^{1} \frac{\left|t^{\frac{\alpha}{k}}-(1-t)^{\frac{\alpha}{k}}\right|}{[t a+(1-t) b]^{2}} \mathrm{~d} t \\
& =2 \int_{0}^{1 / 2} \frac{(1-t)^{\frac{\alpha}{k}}-t^{\frac{\alpha}{k}}}{[t a+(1-t) b]^{2}} \mathrm{~d} t+\int_{0}^{1} \frac{t^{\frac{\alpha}{k}}-(1-t)^{\frac{\alpha}{k}}}{[t a+(1-t) b]^{2}} \mathrm{~d} t \\
& =\frac{1}{b^{2}}\left[I_{1}-\left(\frac{1}{2}\right)^{\frac{\alpha}{k}} \cdot I_{2}+I_{3}-I_{4}\right],
\end{aligned}
$$

with

$$
\begin{aligned}
I_{1}= & \int_{0}^{1}\left(1-\frac{u}{2}\right)^{\frac{\alpha}{k}}\left(1-\frac{b-a}{2 b} u\right)^{-2} \mathrm{~d} u=k F_{1, k}\left(k,-\alpha, 2 k, 2 k ; \frac{1}{2 k}, \frac{b-a}{2 b k}\right) ; \\
I_{2}= & \int_{0}^{1} u^{\frac{\alpha}{k}}\left(1-\frac{b-a}{2 b} u\right)^{-2} \mathrm{~d} u \\
= & k B_{k}(\alpha+k, 1) F_{1, k}\left(\alpha+k, 0,2 k, \alpha+k+1 ; 0, \frac{b-a}{2 b k}\right) ; \\
I_{3}= & \int_{0}^{1} t^{\frac{\alpha}{k}}\left(1-\frac{b-a}{b} t\right)^{-2} \mathrm{~d} t \\
& =k B_{k}(\alpha+k, 1) F_{1, k}\left(\alpha+k, 0,2 k, \alpha+k+1 ; 0, \frac{b-a}{b k}\right) ; \\
I_{4}= & \int_{0}^{1}(1-t)^{\frac{\alpha}{k}}\left(1-\frac{b-a}{b} t\right)^{-2} \mathrm{~d} t=k B_{k}(k, \alpha+k) F_{1, k}\left(k, 0,2 k, \alpha+2 k ; 0, \frac{b-a}{b k}\right),
\end{aligned}
$$


and using the harmonic convexity of $\left|f^{\prime}\right|^{q}$, we have

$$
\begin{aligned}
J= & \int_{0}^{1} \frac{\left|t^{\frac{\alpha}{k}}-(1-t)^{\frac{\alpha}{k}}\right|}{[t a+(1-t) b]^{2}}\left|f^{\prime}\left(\frac{a b}{t a+(1-t) b}\right)\right|^{q} \mathrm{~d} t \\
\leq & \int_{0}^{1} \frac{\left|t^{\frac{\alpha}{k}}-(1-t)^{\frac{\alpha}{k}}\right|}{[t a+(1-t) b]^{2}}\left[(1-t)\left|f^{\prime}(a)\right|^{q}+t\left|f^{\prime}(b)\right|^{q}\right] \mathrm{d} t \\
= & 2 \int_{0}^{1 / 2} \frac{(1-t)^{\frac{\alpha}{k}}-t^{\frac{\alpha}{k}}}{[t a+(1-t) b]^{2}}\left[(1-t)\left|f^{\prime}(a)\right|^{q}+t\left|f^{\prime}(b)\right|^{q}\right] \mathrm{d} t \\
& +\int_{0}^{1} \frac{t^{\frac{\alpha}{k}}-(1-t)^{\frac{\alpha}{k}}}{[t a+(1-t) b]^{2}}\left[(1-t)\left|f^{\prime}(a)\right|^{q}+t\left|f^{\prime}(b)\right|^{q}\right] \mathrm{d} t \\
= & \int_{0}^{1} \frac{\left(1-\frac{u}{2}\right)^{\frac{\alpha}{k}}-\left(\frac{u}{2}\right)^{\frac{\alpha}{k}}}{\left[\frac{u}{2} a+\left(1-\frac{u}{2}\right) b\right]^{2}}\left[\left(1-\frac{u}{2}\right)\left|f^{\prime}(a)\right|^{q}+\frac{u}{2}\left|f^{\prime}(b)\right|^{q}\right] \mathrm{d} u \\
& +\int_{0}^{1} \frac{t^{\frac{\alpha}{k}}-(1-t)^{\frac{\alpha}{k}}}{[t a+(1-t) b]^{2}}\left[(1-t)\left|f^{\prime}(a)\right|^{q}+t\left|f^{\prime}(b)\right|^{q}\right] \mathrm{d} t \\
= & \frac{1}{b^{2}}\left[\left|f^{\prime}(a)\right|^{q}\left(J_{1}-\frac{1}{2^{\alpha / k}} J_{2}+J_{5}-J_{7}\right)\right. \\
& \left.+\left|f^{\prime}(b)\right|^{q}\left(\frac{1}{2} J_{3}-\frac{1}{2^{\alpha / k+1}} J_{4}+J_{6}-J_{8}\right)\right],
\end{aligned}
$$

with

$$
\begin{aligned}
J_{1} & =\int_{0}^{1}\left(1-\frac{1}{2} u\right)^{\frac{\alpha}{k}+1}\left(1-\frac{b-a}{2 b} u\right)^{-2} \mathrm{~d} u=k B_{k}(k, k) F_{1, k}\left(k,-\alpha-k, 2 k, 2 k ; \frac{1}{2 k}, \frac{b-a}{2 b k}\right) ; \\
J_{2} & =\int_{0}^{1} u^{\frac{\alpha}{k}}\left(1-\frac{1}{2} u\right)\left(1-\frac{b-a}{2 b} u\right)^{-2} \mathrm{~d} u \\
& =k B_{k}(\alpha+k, k) F_{1, k}\left(\alpha+k,-k, 2 k, \alpha+2 k ; \frac{1}{2 k}, \frac{b-a}{2 b k}\right) ; \\
J_{3} & =\int_{0}^{1} u\left(1-\frac{1}{2} u\right)^{\frac{\alpha}{k}}\left(1-\frac{b-a}{2 b} u\right)^{-2} \mathrm{~d} u=k B_{k}(2 k, k) F_{1, k}\left(2 k,-\alpha, 2 k, 3 k ; \frac{1}{2 k}, \frac{b-a}{2 b k}\right) ; \\
J_{4} & =\int_{0}^{1} u^{\frac{\alpha}{k}+1}\left(1-\frac{b-a}{2 b} u\right)^{-2} \mathrm{~d} u=k B_{k}(\alpha+2 k, k) F_{1, k}\left(\alpha+2 k, 0,2 k, \alpha+3 k ; 0, \frac{b-a}{2 b k}\right) \\
J_{5} & =\int_{0}^{1} t^{\frac{\alpha}{k}}(1-t)\left(1-\frac{b-a}{b} t\right)^{-2} \mathrm{~d} t=k B_{k}(\alpha+k, 2 k) F_{1, k}\left(\alpha+k, 0,2 k, \alpha+3 k ; 0, \frac{b-a}{b k}\right) ; \\
J_{6} & =\int_{0}^{1} t^{\frac{\alpha}{k}+1}\left(1-\frac{b-a}{b} t\right)^{-2} \mathrm{~d} t=k B_{k}(\alpha+2 k, k) F_{1, k}\left(\alpha+2 k, 0,2 k, \alpha+3 k ; 0, \frac{b-a}{b k}\right) ; \\
J_{7} & =\int_{0}^{1}(1-t)^{\frac{\alpha}{k}+1}\left(1-\frac{b-a}{b} t\right)^{-2} \mathrm{~d} t=k B_{k}(k, \alpha+2 k) F_{1, k}\left(k, 0,2 k, \alpha+3 k ; 0, \frac{b-a}{b k}\right) ; \\
J_{8} & =\int_{0}^{1} t(1-t)^{\frac{\alpha}{k}}\left(1-\frac{b-a}{b} t\right)^{-2} \mathrm{~d} t=k B_{k}(2 k, \alpha+k) F_{1, k}\left(2 k, 0,2 k, \alpha+3 k ; 0, \frac{b-a}{b k}\right)
\end{aligned}
$$

and the proof is complete. 
Theorem 3.7 Assume that $f:[0,1] \rightarrow \mathbb{R}$ is a differentiable function such that $\left|f^{\prime}\right|^{q}$ is a harmonic convex function on $[0,1]$. Then

$$
\left|M_{f}(a, b ; \alpha, k ; g)\right| \leq \frac{a b(b-a)}{2}\left(I^{1-1 / q} J^{1 / q}+K^{1-1 / q} L^{1 / q}+M^{1-1 / q} N^{1 / q}\right),
$$

where I is given by (3.3), J is given by (3.4),

$$
\begin{aligned}
K= & \frac{a}{b^{2}(a+b)}, \\
L \leq & \frac{\left|f^{\prime}(a)\right|^{q}}{2 b^{2}} \cdot k B_{k}(k, k) F_{1, k}\left(k,-k, 2 k, 2 k ; \frac{1}{2 k}, \frac{b-a}{2 b k}\right) \\
& +\frac{\left|f^{\prime}(b)\right|^{q}}{4 b^{2}} \cdot k B_{k}(2 k, k) F_{1, k}\left(2 k, 0,2 k, 3 k ; 0, \frac{b-a}{2 b k}\right), \\
M= & \frac{1}{b(a+b)}
\end{aligned}
$$

and

$$
\begin{aligned}
N \leq & \frac{\left|f^{\prime}(a)\right|^{q}}{2 b^{2}}\left[F_{1, k}\left(k, 0,2 k, 3 k ; 0, \frac{b-a}{b k}\right)-F_{1, k}\left(k,-k, 2 k, 2 k ; \frac{1}{2 k}, \frac{b-a}{2 b k}\right)\right] \\
& +\frac{\left|f^{\prime}(b)\right|^{q}}{2 b^{2}}\left[F_{1, k}\left(2 k, 0,2 k, 3 k ; 0, \frac{b-a}{b k}\right)-\frac{1}{4} F_{1, k}\left(2 k, 0,2 k, 3 k ; 0, \frac{b-a}{b k}\right)\right] .
\end{aligned}
$$

Proof From Lemma 2.3, using the property of modulus and the power-mean inequality, we have

$$
\begin{aligned}
\left|M_{f}(a, b ; \alpha, k ; g)\right| \leq & \frac{a b(b-a)}{2}\left[\int_{0}^{1} \frac{\left|(1-t)^{\frac{\alpha}{k}}-t^{\frac{\alpha}{k}}\right|}{[t a+(1-t) b]^{2}}\left|f^{\prime}\left(\frac{a b}{t a+(1-t) b}\right)\right| \mathrm{d} t\right. \\
& +\int_{0}^{\frac{1}{2}} \frac{1}{[t a+(1-t) b]^{2}}\left|f^{\prime}\left(\frac{a b}{t a+(1-t) b}\right)\right| \mathrm{d} t \\
& \left.+\int_{\frac{1}{2}}^{1} \frac{1}{[t a+(1-t) b]^{2}}\left|f^{\prime}\left(\frac{a b}{t a+(1-t) b}\right)\right| \mathrm{d} t\right] \\
\leq & \frac{a b(b-a)}{2}\left(I^{1-1 / q} J^{1 / q}+K^{1-1 / q} L^{1 / q}+M^{1-1 / q} N^{1 / q}\right),
\end{aligned}
$$

where

$$
K=\int_{0}^{\frac{1}{2}} \frac{1}{[t a+(1-t) b]^{2}} \mathrm{~d} t=\frac{a}{b^{2}(a+b)},
$$

and

$$
\begin{aligned}
L & =\int_{0}^{\frac{1}{2}} \frac{1}{[t a+(1-t) b]^{2}}\left[\left|f^{\prime}\left(\frac{a b}{t a+(1-t) b}\right)\right|\right]^{q} \mathrm{~d} t \\
& \leq \int_{0}^{\frac{1}{2}} \frac{1}{[t a+(1-t) b]^{2}}\left[(1-t)\left|f^{\prime}(a)\right|^{q}+t\left|f^{\prime}(b)\right|^{q}\right] \mathrm{d} t,
\end{aligned}
$$


and using the change of variables, we have

$$
\begin{aligned}
L \leq & \frac{1}{2 b^{2}}\left|f^{\prime}(a)\right|^{q} \int_{0}^{1}\left(1-\frac{1}{2} u\right)\left(1-u \cdot \frac{b-a}{2 b}\right)^{-2} \mathrm{~d} u \\
& +\frac{1}{4 b^{2}}\left|f^{\prime}(b)\right|^{q} \int_{0}^{1} u\left(1-u \cdot \frac{b-a}{2 b}\right)^{-2} \mathrm{~d} u \\
= & \frac{\left|f^{\prime}(a)\right|^{q}}{2 b^{2}} \cdot k B_{k}(k, k) F_{1, k}\left(k,-k, 2 k, 2 k ; \frac{1}{2 k}, \frac{b-a}{2 b k}\right) \\
& +\frac{\left|f^{\prime}(b)\right|^{q}}{4 b^{2}} \cdot k B_{k}(2 k, k) F_{1, k}\left(2 k, 0,2 k, 3 k ; 0, \frac{b-a}{2 b k}\right), \\
M= & \int_{\frac{1}{2}}^{1} \frac{1}{[t a+(1-t) b]^{2}} \mathrm{~d} t=\frac{1}{b(a+b)},
\end{aligned}
$$

and

$$
\begin{aligned}
N= & \int_{\frac{1}{2}}^{1} \frac{1}{[t a+(1-t) b]^{2}}\left[\left|f^{\prime}\left(\frac{a b}{t a+(1-t) b}\right)\right|\right]^{q} \mathrm{~d} t \\
= & \int_{0}^{1} \frac{1}{[t a+(1-t) b]^{2}}\left[\left|f^{\prime}\left(\frac{a b}{t a+(1-t) b}\right)\right|\right]^{q} \mathrm{~d} t \\
& -\int_{0}^{\frac{1}{2}} \frac{1}{[t a+(1-t) b]^{2}}\left[\left|f^{\prime}\left(\frac{a b}{t a+(1-t) b}\right)\right|\right]^{q} \mathrm{~d} t \\
\leq & \frac{1}{b^{2}} \int_{0}^{1}\left(1-t \cdot \frac{b-a}{b}\right)^{-2}\left[(1-t)\left|f^{\prime}(a)\right|^{q}+t\left|f^{\prime}(b)\right|^{q}\right] \mathrm{d} t \\
& -\frac{1}{b^{2}} \int_{0}^{\frac{1}{2}}\left(1-t \cdot \frac{b-a}{b}\right)^{-2}\left[(1-t)\left|f^{\prime}(a)\right|^{q}+t\left|f^{\prime}(b)\right|^{q}\right] \mathrm{d} t \\
= & \frac{\left|f^{\prime}(a)\right|^{q}}{b^{2}}\left[\int_{0}^{1}(1-t)\left(1-t \cdot \frac{b-a}{b}\right)^{-2} \mathrm{~d} t-\int_{0}^{\frac{1}{2}}(1-t)\left(1-t \cdot \frac{b-a}{b}\right)^{-2} \mathrm{~d} t\right] \\
& +\frac{\left|f^{\prime}(b)\right|^{q}}{b^{2}}\left[\int_{0}^{1} t\left(1-t \cdot \frac{b-a}{b}\right)^{-2} \mathrm{~d} t-\int_{0}^{\frac{1}{2}} t\left(1-t \cdot \frac{b-a}{b}\right)^{-2} \mathrm{~d} t\right] \\
= & \frac{\left|f^{\prime}(a)\right|^{q}}{2 b^{2}}\left[F_{1, k}\left(k, 0,2 k, 3 k ; 0, \frac{b-a}{b k}\right)-F_{1, k}\left(k,-k, 2 k, 2 k ; \frac{1}{2 k}, \frac{b-a}{b k}\right)\right] \\
& +\frac{\left|f^{\prime}(b)\right|^{q}}{2 b^{2}}\left[F_{1, k}\left(2 k, 0,2 k, 3 k ; 0, \frac{b-a}{b k}\right)-\frac{1}{4} F_{1, k}\left(2 k, 0,2 k, 3 k ; 0, \frac{b-a}{2 b k}\right)\right] .
\end{aligned}
$$

This completes the proof.

\section{Conclusion}

A new refinement of Hermite-Hadamard's inequality via $k$-Riemann-Liouville fractional integrals is obtained. We have derived two new $k$-fractional integral identities. Utilizing these identities, we have derived some new $k$-fractional bounds which involve $k$-Appell's hypergeometric functions via the functions which have the harmonic convexity property. It is expected that the ideas and techniques of this article may be useful for future research. 
Competing interests

The authors declare that they have no competing interests.

\section{Authors' contributions}

MUA, MAN, MVM and KIN worked jointly. All the authors read and approved the final manuscript.

\section{Author details}

${ }^{1}$ Department of Mathematics, Government College University, Faisalabad, Pakistan. ${ }^{2}$ Department of Mathematics, King Saud University, Riyadh, Saudi Arabia. ${ }^{3}$ Department of Mathematics, COMSATS Institute of Information Technology, Islamabad, Pakistan. ${ }^{4}$ Department scientific-methodical sessions, Romanian Mathematical Society-branch Bucharest, Academy Street no. 14, Bucharest, 010014, Romania.

\section{Acknowledgements}

Authors are thankful to anonymous referees for their valuable comments and suggestions. Authors are pleased to acknowledge the support of Distinguished Scientist Fellowship Program (DSFP), King Saud University, Riyadh, Saudi Arabia.

\section{Publisher's Note}

Springer Nature remains neutral with regard to jurisdictional claims in published maps and institutional affiliations.

Received: 15 February 2017 Accepted: 2 May 2017 Published online: 18 May 2017

\section{References}

1. Breckner, WW: Stetigkeitsaussagen für eine Klasse verallgemeinerter konvexer funktionen in topologischen linearen Raumen. Pupl. Inst. Math. 23, 13-20 (1978)

2. Cristescu, G, Lupsa, L: Non-connected Convexities and Applications. Kluwer Academic Publishers, Dordrecht, Holland (2002)

3. Cristescu, G, Noor, MA, Awan, MU: Bounds of the second degree cumulative frontier gaps of functions with generalized convexity. Carpath. J. Math. 31(2), 173-180 (2015)

4. Dragomir, SS: Inequalities of Hermite-Hadamard type for $h$-convex functions on linear spaces. RGMIA Res. Rep. Coll., Article ID 72 (2013)

5. Dragomir, SS, Pečarić, J, Persson, LE: Some inequalities of Hadamard type. Soochow J. Math. 21, 335-341 (1995)

6. Dragomir, SS, Pearce, CEM: Selected Topics on Hermite-Hadamard Inequalities and Applications. Victoria University Australia (2000)

7. Godunova, EK, Levin, VI: Neravenstva dlja funkcii sirokogo klassa, soderzascego vypuklye, monotonnye i nekotorye drugie vidy funkii. Vycislitel. Mat. i. Fiz. Mezvuzov. Sb. Nauc. Trudov, MGPI, Moskva., 138-142 (1985) (in Russian)

8. Iscan, I: Hermite-Hadamard type inequalities for harmonically convex functions. Hacet. J. Math. Stat. 43(6), 935-942 (2014)

9. Noor, MA, Noor, Kl, Awan, MU: Integral inequalities for coordinated harmonically convex functions. Complex Var. Elliptic Equ. 60(6), 776-786 (2015)

10. Noor, MA, Noor, KI, Awan, MU, Costache, S: Some integral inequalities for harmonically $h$-convex functions. UPB Sci. Bull., Series A. 77(1), 5-16 (2015)

11. Shi, H-N, Zhang, J: Some new judgement theorems of Schur geometric and Schur harmonic convexities for a class of symmetric functions. J. Inequal. Appl. 2013, 527 (2013)

12. Varočanec, S: On h-convexity. J. Math. Anal. Appl. 326, 303-311 (2007)

13. Iscan, I: Hermite-Hadamard and Simpson-like type inequalities for differentiable harmonically convex functions. J. Math. 2014, Article ID 346305 (2014)

14. Mihai, MV, Noor, MA, Noor, Kl, Awan, MU: Some integral inequalities for $h$-convex functions involving hypergeometric functions. Appl. Math. Comput. 252(1), 257-262 (2015)

15. Park, J: Hermite-Hadamard-like and Simpson-like type inequalities for harmonically convex functions. Int. J. Math. Anal. 8(27), 1321-1337 (2014)

16. Antczak, T, Pitea, A: Parametric approach to multitime multiobjective fractional variational problems under $(F, \rho)$-convexity. Optim. Control Appl. Methods 37(5), 831-847 (2016)

17. Pitea, A, Antczak, T: Proper efficiency and duality for a new class of nonconvex multitime multiobjective variational problems. J. Inequal. Appl. 2014, Art. No. 333 (2014)

18. Pitea, A, Postolache, M: Duality theorems for a new class of multitime multiobjective variational problems. J. Glob. Optim. 54(1), 47-58 (2012)

19. Pitea, A, Postolache, M: Minimization of vectors of curvilinear functionals on the second order jet bundle. Necessary conditions. Optim. Lett. 6(3), 459-470 (2012)

20. Pitea, A, Postolache, M: Minimization of vectors of curvilinear functionals on the second order jet bundle. Sufficient efficiency conditions. Optim. Lett. 6(8), 1657-1669 (2012)

21. Khattri, SK: Three proofs of the inequality $e<\left(1+\frac{1}{n}\right)^{n+0.5}$. Am. Math. Mon. 117(3), 273-277 (2010)

22. Kilbas, A, Srivastava, HM, Trujillo, Jj: Theory and Applications of Fractional Differential Equations. Elsevier, Amsterdam, Netherlands (2006)

23. Sarikaya, MZ, Set, E, Yaldiz, H, Basak, N: Hermite-Hadamard's inequalities for fractional integrals and related fractional inequalities. Math. Comput. Model. 57, 2403-2407 (2013)

24. Diaz, R: On hypergeometric functions and Pochhammer k-symbol. Divulg. Mat. 2, 179-192 (2007)

25. Mubeen, S, Habibullah, GM: k-fractional integrals and applications. Int. J. Contemp. Math. Sci. 7(2), 89-94 (2012)

26. Sarikaya, MZ, Karaca, A: On the k-Riemann-Liouville fractional integral and applications. Int. J. Math. Stat. 1(3), 33-43 (2014) 
27. Mubeen, S, Iqbal, S, Rahman, G: Contiguous function relations and an integral representation for Appell $k$-series $F_{1, k}$. Inter. J. Math. Research 4(2), 53-63 (2015)

28. Set, E, Iscan, I, Zehir, F: On some new inequalities of Hermite-Hadamard type involving harmonically convex functions via fractional integrals. Konuralp J. Math. 3(1), 42-55 (2015)

Submit your manuscript to a SpringerOpen ${ }^{\circ}$ journal and benefit from:

- Convenient online submission

- Rigorous peer review

- Immediate publication on acceptance

- Open access: articles freely available online

- High visibility within the field

- Retaining the copyright to your article

Submit your next manuscript at $\boldsymbol{s p r i n g e r o p e n . c o m ~}$ 